\title{
Gross and Histopathological Alterations in Experimental Trypanosoma Evansi Infection in Donkeys and the Effect of Isometamidium Chloride Treatment
}

\author{
Garba $\mathrm{UM}^{*}$, Sackey $\mathrm{AKB}^{2}$, Lawal $\mathrm{AI}^{3}$, Esievo $\mathrm{KAN}^{4}$, Bisalla $\mathrm{M}^{4}$ and Sambo JS \\ ${ }^{1}$ Veterinary Clinic, Equitation Dept, Nigerian Defence Academy, Kaduna, Nigeria \\ ${ }^{2}$ Department of Veterinary Medicine, Faculty of Veterinary Medicine, Ahmadu Bello University Zaria, Nigeria \\ ${ }^{3}$ Department of Veterinary Parasitology and Entomology, Faculty of Veterinary Medicine, Ahmadu Bello University \\ Zaria, Nigeria \\ ${ }^{4}$ Department of Veterinary Pathology, Faculty of Veterinary Medicine, Ahmadu Bello University Zaria, Nigeria
}

*Corresponding author: Garba UM, Veterinary Clinic, Equitation Department, Nigerian Defense Academy, P.M.B. 2109, Kaduna, Nigeria, Tel: +2348034524912, E-mail: garbaum@yahoo.com

Citation: Garba UM, Sackey AKB, Lawal AI, Esievo KAN, Bisalla M, et al. (2016) Gross and Histopathological Alterations in Experimental Trypanosoma Evansi Infection in Donkeys and the Effect of Isometamidium Chloride Treatment. J Vet Sci Animl Husb 5(1): 104. doi: 10.15744/2348-9790.5.104

Received Date: November 14, 2016 Accepted Date: February 24, 2017 Published Date: February 27, 2017

\begin{abstract}
Trypanosoma evansi (T. evansi) infection causes wasting and fatal animal trypanosomosis. This study was aimed at determining the gross and histopathological alterations in donkeys experimentally infected with T. evansi and the effect of isometamidium chloride treatment. Apparently healthy donkeys $(\mathrm{N}=18)$ of mixed sexes were randomly assigned to 3 groups; $\mathrm{A}_{1}$ (Infected-untreated), $\mathrm{A}_{2}$ (Infected, isometamidium-treated) and B (Uninfected, control) of six animals each. Each animal in infected groups had about $2.0 \times 10^{6}$ T. evansi injected through the jugular vein. Parasitaemia levels were evaluated using HCT and Mice Inoculation Test (support test). Gross and histopathological examinations were also conducted post-infection and post-treatment. Results, showed chronicstage relapsed parasitaemia in infected-untreated group and much later in isometamidium-treated animals. Grossly, acute lesions were severe lungs congestion and frothy exudates in the trachea/bronchi while the chronic lesions were moderate gray hepatisation, hydrothorax, hydroperitoneum, serous atrophies of fats and mild adherence of renal capsule to the cortex. Histopathological lesions were chronic and involved the lungs (moderate to severe forms of congestion and mononuclear cellular infiltrations), spleen (severe forms of haemosiderosis and lymphocytes depletion at germinal centres) and liver (severe form of congestion, moderate focal necrosis and mononuclear cellular infiltrations). Isometamidium chloride-treated animals showed only moderate level of mononuclear cellular infiltrations in lungs while in spleen mild levels of haemosiderosis and lymphocytes depletion at germinal centres were the histopathological features. In conclusion, there is parasite relapse, the gross and histopathological lesions in experimental T. evansi infection in donkeys are pronounced in acute and chronic stages of infection manifesting as severe to moderate lesions in thoracic and peritoneal cavities, lungs, spleen, heart, kidneys and liver of animals that do not get treatment. Donkeys treated with isometamidium chloride, show relapsed parasitaemia, mild to moderate histopathological lesions in lungs and spleen with no gross lesions in chronic stage.
\end{abstract}

Keywords: Alterations; Donkeys; Gross; Histopathological; Isometamidium chloride, Trypanosoma evansi

List of Abbreviations : AIT: Animal inoculation test; EDTA+K3: Tripotassium salt of ethylene diamine tetra-acetate; FAO: Food and Agricultural Organisation; HCT: Haematocrit centrifugation technique; H-E: Haematoxylin-eosin; ILRI: International Livestock Research Institute; Kg: Kilogramme; MIT: Mice inoculation tests; mL: Milliliters; OIE: Office International des Epizootes; PI: Postinfection; PT: Post-treatment; SAS: System analytical statistics; T. evansi: Trypanosoma evansi; UK: United Kingdom; USA: United States of America; WHO: World Health Organisation

\section{Introduction}

Trypanosoma evansi (T. evansi) infection causes a progressively wasting and ultimately fatal animal trypanosomosis (surra) mainly in camels but cattle, horses, donkeys, zebra, mules, dogs and pigs are also affected [1,2]. The causative parasite, T. evansi is a haemo - protozoan of the genus; Trypanosoma [3,4]. It is transmitted mechanically from an infected animal to another susceptible animal by biting flies (vectors) mainly the Tabanus spp but Stomoxys sp, Haematopota sp, Lyperosia sp as well as Chrysops sp [5]. Trans - placental transmission route has also been reported in donkeys [6]. In Nigeria, the chronic form of natural clinical surra is reported to occur in donkeys [7]. However, the associated pathologies are not sufficiently reported to provide additional guide for diagnosis and supportive therapy to ameliorate specific pathological alterations. 
The parasite is widespread in the tropical and subtropical areas of the world such as Asia, Africa, Middle East, Central and South America causing great economic losses due to reduced traction power and death of thousands of affected animals annually [8,9]. Isometamidium chloride (Samorin ${ }^{\oplus}$, May and Baker, Nigeria; Securidium ${ }^{\oplus}$, Laprovet, France) belongs to the same chemical group as homidium [10], the group of the phenanthridine aromatic amidine compounds. It is a dark red powder, producing a brownish red solution in water for injection as $1 \%$ or $2 \%$ concentration. It is an antitrypanosomal drug with curative and prophylactic actions, registered for use principally in cattle, sheep, goat, camel, horse and dog with efficacies against Trypanosoma congolense, Trypanosoma vivax, Trypanosoma brucei and Trypanosoma evansi at the standard dose rates of $0.25-0.5 \mathrm{mg} / \mathrm{kg}$ body weight, intramuscularly to achieve cure and a sanative dose of $1 \mathrm{mg} / \mathrm{kg}$ body weight, intramuscularly for prophylaxis [11]. Local tissue reaction occurs in cattle and diminazene is recommended as the sanitive pair for treatment of relapse from isometamidium chloride therapy [12]. The reported protection duration of isometamidium is as long as 5 months and has been used in many parts of the world in the treatment of trypanosomosis in animals as cited by Delespaux and De Koning and Karaye [13-15].

The aim of this study was to determine the gross and histopathological alterations in donkeys experimentally infected with Trypanosoma evansi and the effect of Isometamidium chloride treatment. In this experiment, donkeys experimentally infected with Trypanosoma evansi were evaluated for parasitaemia Levels (using HCT and mice inoculation test) post-infection and reevaluated post - treatment. At postmortem, the thoracic and abdominal cavities, liver, kidney, spleen, heart and skeletal muscle, brain, testes and ovary of experimental donkeys were examined for gross lesions. Processed tissue sections were microscopically examined for histopathological alterations.

\section{Materials and Methods}

\section{Experimental animals}

Apparently healthy donkeys $(\mathrm{N}=18)$, comprising of 9 males and 9 females were used for this experiment. At point of purchase in International Livestock Market, Maigatari, Jigawa State, Nigeria, the ages of the donkeys were estimated to be 11 to 15 months using features of incisor teeth, body condition scores ranged from 4 to 6 (Medium; balanced body frame and body cover) using the nine point scale system described by Pearson and Ouassat [16-18]. Pre-infection, body weight range of the donkeys was $62.0 \mathrm{~kg}$ to $69.8 \mathrm{~kg}$ as determined using sling scale between the hours of $06.00 \mathrm{am}$ and $08.00 \mathrm{am}$ to minimize the influences of gut-fill and hydration on body weight value $[19,20]$.

Four (4) healthy and haemoparasites-free, adult albino Wistar rats, with body weight range of $200 \mathrm{~g}$ - $220 \mathrm{~g}$ were used for the propagation of T. evansi to obtain sufficient inoculum for experimental infection of donkeys.

Healthy, haemoparasites-free, adult Wistar mice with body weight range of $14 \mathrm{~g}-16 \mathrm{~g}$ were used in Mice inoculation tests (MIT) conducted for the experimental donkeys. The MIT was used as support test for Haematocrit Centrifugation Test in the evaluation of the donkeys' haemoparasites-free status (pre-infection), parasitaemia monitoring (post-infection and post-treatment) using two mice per donkey in each test as recommended by OIE [21]. The rats and mice were sourced from Animal Laboratory, Faculty of Pharmaceutical Sciences, Ahmadu Bello University, Zaria, Nigeria.

\section{Management and pre-infection evaluation of experimental animals}

Housing and acclimatization: The experimental animals were examined for signs of disease and external parasites infestations. Topical sweat-resistant fly-repellant spray (Endure ${ }^{\oplus}$, Farnam co. inc., USA) was applied on the animals to keep flies away as they were moved into prepared, compartmentalized, fly-proof experimental animal pen. Wood shaving was used as bedding material on the floor of the pen to prevent damp. Faecal droppings were mucked three times daily and the bedding was changed as soon as the old one was becoming damp. The experimental animals were acclimatized for 14 days [22].

Identification and grouping of animals: Aluminum plate neck-tags (about $5 \mathrm{~g}$ each) with engraved serial numbers were assigned to the donkeys at random for identification. A sample frame of each gender was prepared and six (6) animals (3 males and 3 females) were assigned to each of groups $\mathrm{A}_{1}, \mathrm{~A}_{2}$ and $\mathrm{B}$ at random [23].

The rats (for parasite propagation) and mice (for animal inoculation tests) were caged separately. The mice were accommodated in cages according to the group of donkeys whose blood was used to sub-inoculate them during mice inoculation test. Permanent marker pen was further used to apply marks on one particular body part of every set of two mice that represented a particular donkey in a group. Therefore, every set of two mice had their marks on the same body part reflecting the donkey whose blood was used to inoculate them.

Feeding of experimental animals: Feed was provided daily equivalent to $5 \%$ of the mean body weight of the donkeys. It comprised of 4 parts of mixed sorghum/maize stovers and Rhodes grass, and 1 part of groundnuts hay (4: 1 ratio). Concentrate feed consisted of a mixture of crushed sorghum grain $(500 \mathrm{~g})$, dried groundnut cake (130g) and maize/wheat bran (2.5kg) per 200-300kg bd wt of donkeys recommended by Aganga., et al. [24]. Salt lick blocks, rich in dicalcium phosphate and clean drinking water were provided ad-libitum. 
The albino rats and Wister mice were maintained in separate, labeled cages and were provided with commercially obtained pelletised feed in feeders and clean drinking water in sipper tubes ad libitum.

Pre-infection evaluation: As part of procedures to further determine and ensure the well-being of the experimental animals before they were infected with $T$. evansi, about $5 \mathrm{~g}$ of faecal sample was collected from the rectum of each animal into labeled polyethylene bag and examined for gastro-intestinal parasites using floatation and sedimentation methods as reported by Charles [25]. The animals were dewormed using fenbendazole bolus (Fenacure ${ }^{\varpi}$, Ashish Life Sciences PVT ltd, India) at the dose rate of $10 \mathrm{mg} / \mathrm{kg}$ bd wt orally, once [12]. Three days after the deworming, another set of faecal samples were collected and examined to ensure that animals were free from worms. Four (4) $\mathrm{mL}$ of blood was collected from each donkey through the jugular vein using $5 \mathrm{~mL}$ syringe and 18G needle and examined as follows: Three milliliters of the collected blood sample was anticoagulated in sample bottle containing tripotassium salt of ethylene diamine tetra-acetate $\left(\right.$ EDTA $\left.+\mathrm{K}_{3}\right) 1 \mathrm{mg} / \mathrm{mL}$ of whole blood while the balance of $1 \mathrm{~mL}$ blood was anticoagulated in sodium heparin-coated $1 \mathrm{~mL}$ sterile disposable syringe [26]. From each of the EDTA - anticoagulated blood sample, 2 preparations of Wet Blood Film, HCT and Giemsa Stained thin blood smear were made and examined immediately for haemoparasites infections. Pre-infection PCV was also evaluated from the prepared HCT tube [27]. An average of 20 microscope fields of each stained thin blood smear slides were examined for haemoparasites [26]. As a support test for direct examinations of the donkeys' blood for parasites, Animal Inoculation Test (AIT) was performed using the $1 \mathrm{~mL}$ heparinised blood sample from each donkey. Two mice were used to test the blood of each experimental donkey as recommended by OIE [21] with each mice receiving $0.5 \mathrm{~mL}$ of the heparinised blood through intra-peritoneal inoculation using $1 \mathrm{~mL}$ sterile disposable syringe with $29 \mathrm{GX}{ }^{1 / 2}$ inch needle [22]. The inoculated mice were monitored for haemoparasites on HCT and Wet Film using their tail-tip-blood, examined at 48 hours intervals for 14 days [28] before the donkeys were experimentally infected with T. evansi. An average of 20 microscope fields of each prepared slide was examined each time. While next stages of the experiment continued, the mice remained under observation to day 60 post-inoculation.

Propagation of Trypanosoma evansi: Trypanosoma evansi originally isolated from camel in Sokoto (Sokoto isolate) and maintained in Wistar rats in the Department of Veterinary Parasitology and Entomology, Ahmadu Bello University, Zaria, was used in this experiment.

One milliliter of parasitaemic blood collected via ocular vein from an infected Wistar rat was heparinised and diluted with $1 \mathrm{~mL}$ phosphate buffered saline glucose solution to obtain $2 \mathrm{~mL}$ of inoculum. About $0.5 \mathrm{~mL}$ of the prepared parasitaemic inoculum was injected into each of a set of 4 donor rats, intra-peritoneally using sterile $1 \mathrm{~mL}$ disposable syringe, $25 \mathrm{G}_{\mathrm{x}} 1 \frac{11 / 2}{2}$ inch needle [28,22] to increase parasites yield.

Subsequently, the donor rats were monitored for parasitaemia levels at 48 hours intervals using HCT. On day 12 post-inoculation, the 4 rats had attained between +2 to +4 parasitaemia levels. The donor rats were then bled into a sterile conical flask and the pooled parasitaemic blood was anti-coagulated with heparin sodium (20 Units/mL of blood). The blood was diluted with phosphate buffered saline glucose solution to obtain an inoculum containing about $1.0 \times 10^{6}$ T. evansi /mL of blood [28].

Experimental infection of animals: Two $\mathrm{mL}$ of the prepared inoculum containing about $2.0 \times 10^{6}$ viable T. evansi was immediately injected into each of the experimental donkeys in groups $A_{1}$ and $A_{2}$ through the jugular vein and the day was considered as day 0 of infection. Group B animals remained as Un-infected control.

Post-infection parasitaemia evaluation: The infected donkeys were subsequently monitored for parasitaemia levels post-infection (PI) from ear vein blood collected and examined on HCT and Giemsa-stained thin blood smears [19,21]. Levels of parasitemia were scored using $+1,+2,+3$ and +4 representing $1-5,6-10,11-20$ and $>20$ parasites per microscopic field respectively [29]. From day 1 to day 7 PI, animals were evaluated for parasitaemia levels once daily. Further evaluation from day 8 to day 28 PI, was performed twice weekly.

Treatment of infected animals: On day $28 \mathrm{PI}$, all infected animals (groups $\mathrm{A}_{1}$ and $\mathrm{A}_{2}$ ) had attained +4 parasitaemia level on HCT examination. Immediately, the animals in group A2 were treated with isometamidium chloride (Securidium ${ }^{\oplus}$, Laprovet, France) $1 \%$ solution at the dose rate of $0.5 \mathrm{mg} / \mathrm{kg}$ body weight using deep intramuscular route once. Group $\mathrm{A}_{1}$ was maintained as infected but untreated while the group B animals were un-infected, untreated (Control). Treatment day was considered as day 0 of treatment.

Gross pathological evaluation: Two infected animals died from group $\mathrm{A}_{1}$, first on days 11 and another one on day 28 PI. The carcasses were examined at post-mortem while still fresh for gross lesions resulting from T. evansi infection especially the lesions in the thoracic and peritoneal cavities, liver, kidney, spleen, heart and gluteal muscle, brain, testes and ovary. At termination of experiment on day 176 PI, one animal was picked at random from each of the 3 groups, the animals were exsanguinated and examined at post-mortem as reported by Wolfensohn and Lloyd [22]. The observations were compared to determine the effects of treatments, non-treatment with normal organs of the control animal.

Histopathological evaluation: Tissue samples were collected from the liver, kidney, spleen, heart, gluteal muscles, brain, testes and ovaries during post-mortem examinations. The samples were fixed in $10 \%$ formaldehyde except brain, testes and ovary samples which were fixed in Bouin's solution. All samples were stored in labeled plastic sample bottles until processed. At processing, 
the tissues were embedded in paraffin wax, cut at $5 \mu$ thickness, mounted on microscope slides and stained with haematoxylineosin (H-E) as described by Drury and Wallington [30]. The prepared sections were examined under light microscope (Zeiss Microscope ${ }^{\oplus}$, Olympus Optical Co. 1td, Japan) using x200 and x400 objectives for histopathological changes.

The coloured images of organs and tissue sections revealing histopathological lesions were captured using digital camera (Model ES30, Samsung, U.K.)

\section{Data Analysis}

Means of parasitaemia levels (post-infection and post-treatment) for various experimental groups were determined using System Analytical Statistics (SAS) corporation - 2010. The means of parasitaemia levels are presented on graphs using Microsoft Excel 2010 to illustrate the trend in levels changes. The severities of gross and histopathological findings from organs were scored as Mild (+), Moderate $(++)$, Severe $(+++)$ lesion. Insignificant gross or histopathological findings were scored as negative $(-)$ and presented in tables.

\section{Results}

\section{Findings from pre-infection evaluation of experimental animals}

The first pre-infection examination of faecal samples of the animals showed: Strongyle eggs load of +1 in $6 / 24(25 \%)$ and +2 in $4 / 24$ (16.7\%). Examination result for faecal samples collected after deworming was negative for gastro-intestinal parasites. Blood samples examined were also negative for haemoparasites (including Trypanosoma species). Observed packed cell volume was 35\% - $46 \%$, normal range is $32 \%-48 \%$ as reported by Wosu (2002).

\section{Post-infection mean levels of parasitaemia in groups of donkeys}

Both groups $\mathrm{A}_{1}$ and $\mathrm{A}_{2}$ animals infected with T. evansi showed parasitaemia for the first time on day 3 post-infection (PI). Peaks of mean parasitaemia counts were observed on day $7\left(A_{1}=13\right.$ parasites per microscope field, $A_{2}=10 /$ field $)$ and day $18\left(A_{1}=20 /\right.$ field, $A_{2}=19 /$ field). Interval between peaks of parasitaemia was 11 days. Observed increases in mean parasitaemia levels between first peak on day 7 and second peak on day 18 PI were; group $A_{1}=7 / 13(53.8 \%)$ and $A_{2}=9 / 10(90 \%)$. During a third rising waves of parasitaemia (not yet at peak) on day $28 \mathrm{PI}$ (mean parasitaemia in $\mathrm{A}_{1}=10 /$ field and $\mathrm{A}_{2}=16 /$ field), group $\mathrm{A}_{2}$ animals were treated with isometamidium. The treatment day (day $28 \mathrm{PI}$ ) was considered day 0 of treatment (arrowed on X-axis of Figure1).

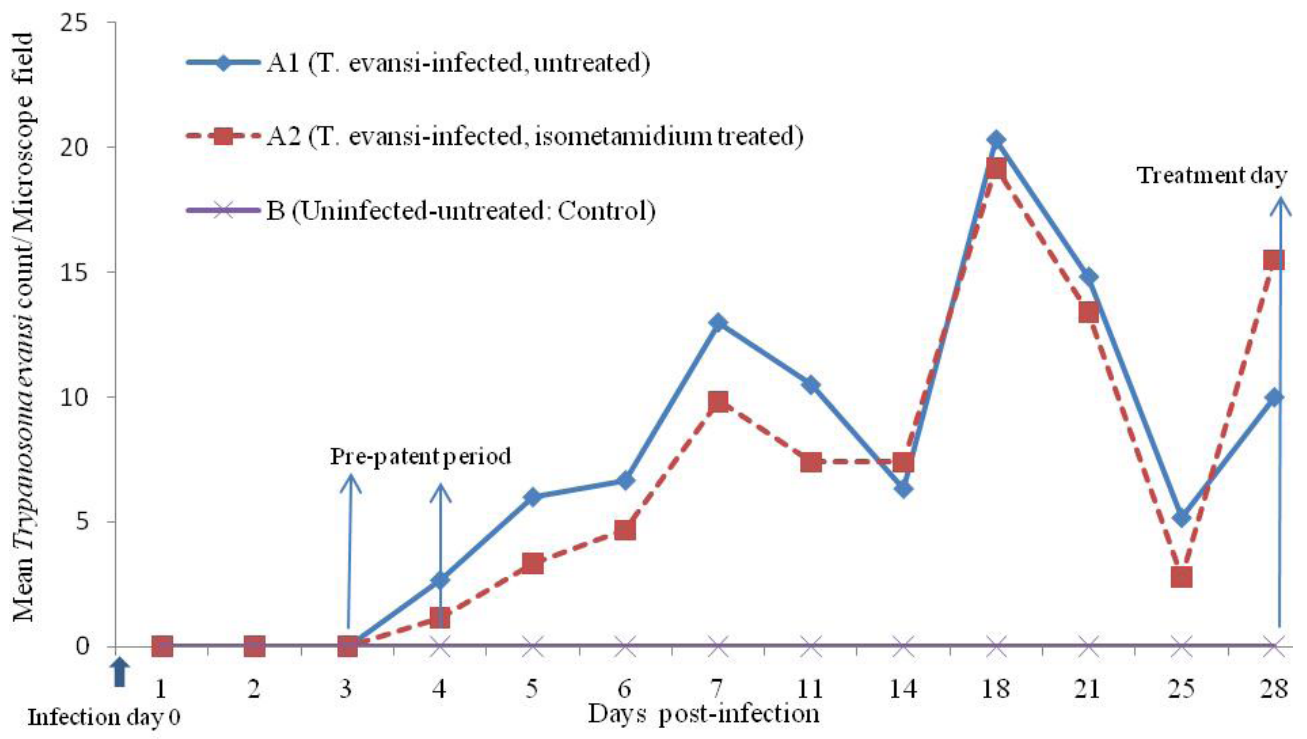

Figure 1: Mean levels of parasitaemia in donkey experimentally infected with Trypanosoma evansi

\section{Post-treatment mean levels of parasitaemia in groups of donkeys}

Group A (Infected-untreated): The infected-untreated group $A_{1}$ animals, maintained an undulating wave of parasitaemia which gradually declined in values from 12/field on day 5 to 2/field on day 58 PI (decline=10/12; 83.3\%) over 53 days period, until the group was aparasitaemic on on day 100 PI (equivalent to day 72 PT; Figure 2) using direct HCT examination. However, when blood samples from the donkeys were subjected to mice inoculation test (MIT) on day 110 PI and the mice were monitored for parasitaemia, positive parasitaemias of +3 and $+4 /$ fields were observed in the mice using HCT. By day 15 post-inoculation, all of the inoculated mice had died of the infection. This means that latent infection existed in the donkeys as at day 100 PI which was not detected by direct examination of the donkeys' blood using HCT. 


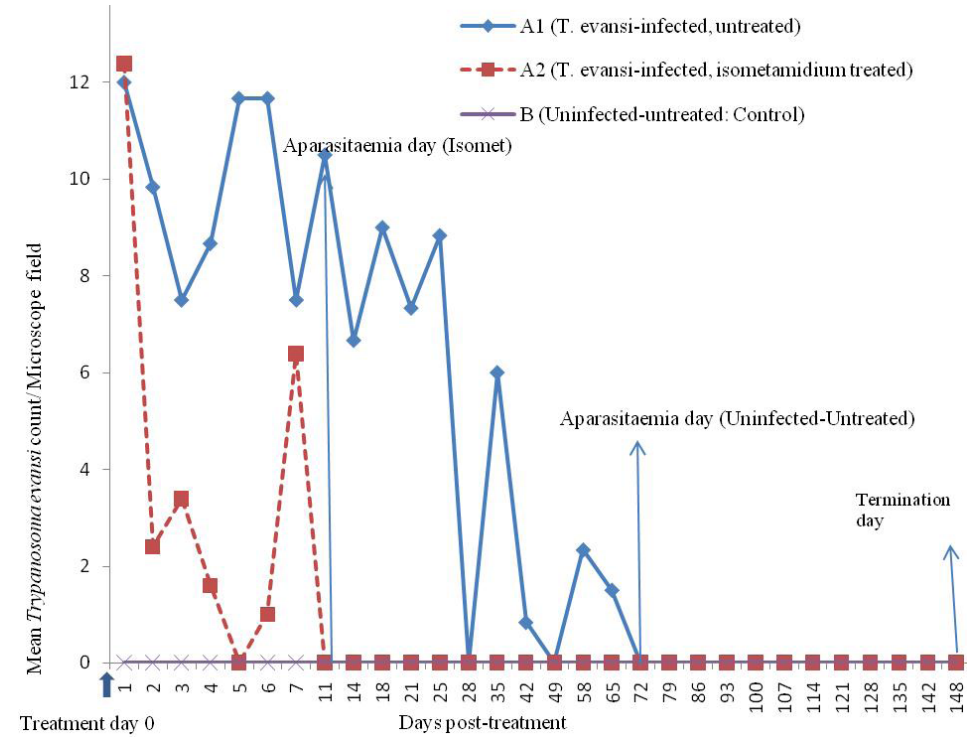

Figure 2: Mean levels of Trypanosoma evansi observed in experimentally infected donkeys post-treatment

Group $\mathbf{A}_{2}$ (Infected, Isometamidium-treated): The T. evansi-infected, isometamidium chloride-treated group of animals showed sharp drop in mean parasitaemia on direct HCT test from approximately 16/field on day 28 PI (Figure 1) that is day 0 PT (Figure 2) to about 2/field on day 2 PT giving a decline from 16 to 2 , that is $14 / 16$ (87.5\%) within 2 days PT. Parasitaemia was zero on direct HCT on day 11 PT. Mice inoculation tests (MIT) on days 21 and 50 were negative but showed positive result on day 100 PT in 6/10 (60\%) of mice used representing $3 / 5(60 \%)$ of donkeys in the group. The MIT showed that there was no detectable parasitaemia within 50 days PT but on day $100 \mathrm{PT}$, latent parasitaemia status was detected in 3/5 (60\%) of the donkeys treated with isometamidium. The inoculated mice showed only 1 parasite (1+) per microscope field both on Wet Film and HCT during 48 days period of post-inoculation observation of the mice. The remaining 4 mice (representing 2 donkeys in the group) were negative both on WF and HCT for the same period of observation.

\section{Gross lesions in donkeys experimentally infected with Trypanosoma evansi}

Group A (Infected-untreated): In the early stage (first 28 days post-infection; PI), the gross lesion observed in the carcasses of infected but untreated animals at postmortem were limited to the lungs and comprised of severe frothy exudates in the trachea and bronchi as well as congestion of lungs. In the later stage (chronic, day $176 \mathrm{PI}$ ), the severity of frothy exudates and congestion in lungs reduced to the mild form but in addition, moderate forms of gray hepatisation, hydrothorax, hydroperitoneum, serous atrophies of fat (mesenteric, renal and coronary fats) were present. Mild adherence of renal capsule to the renal cortex was also observed chronically. (Table.1, Plates Ib \& IIb).

Group $\mathbf{A}_{2}$ (Infected, isometamidium-treated): The animals treated with Isometamidium chloride, in the later (chronic stage, day 148 PT) only manifested mild forms of frothy exudates in trachea and bronchi as well as lungs congestion as prominent gross lesions. All other gross lesions observed chronically in the untreated group were not significant in the organs of Isometamidiumtreated animals (Table 1, Plate Ic)

\begin{tabular}{|c|c|c|c|c|c|}
\hline \multirow{3}{*}{$\begin{array}{l}\text { Organs/cavities } \\
\text { with lesions }\end{array}$} & \multirow{3}{*}{ Lesions observed } & \multicolumn{4}{|c|}{ Severity of lesions in experimental animal groups } \\
\hline & & \multicolumn{2}{|c|}{ Group $A_{1}$ (Infected- untreated) } & \multirow{2}{*}{$\begin{array}{c}\text { Group } A_{2} \text { (Isometa- } \\
\text { midium- treated) }\end{array}$} & \multirow{2}{*}{$\begin{array}{c}\begin{array}{c}\text { Group B (Unin- } \\
\text { fected-untreated) }\end{array} \\
\text { Day176 PI }\end{array}$} \\
\hline & & $\begin{array}{l}\text { Post-infection } \\
\text { Days } 11 \text { and } 28\end{array}$ & $\begin{array}{l}\text { Post-infection } \\
\text { Day176 }\end{array}$ & & \\
\hline \multirow[t]{3}{*}{ Lungs } & $\begin{array}{l}\text {-Frothy exudates in trachea and } \\
\text { bronchi }\end{array}$ & +++ & $+($ Plate. Ib) & + & -- (Plate. Ia) \\
\hline & -Congestion & +++ & + (Plate. Ib) & + (Plate. Ic) & - \\
\hline & -Gray hepatisation & - & ++ (Plate. Ib) & - & - \\
\hline Thoracic cavity & -Hydrothorax (300ml) & - & ++ & - & - \\
\hline Peritoneal cavity & -Hydroperitoneum (straw coloured) & - & ++ & - & - \\
\hline $\begin{array}{l}\text { Mesenteric, renal } \\
\text { and coronary fat }\end{array}$ & -Serous atrophies of fats & - & ++ (Plate. IIb) & - & - (Plate. IIa) \\
\hline Kidneys & $\begin{array}{c}\text {-Adherence of renal capsule to the } \\
\text { renal cortex }\end{array}$ & - & + & - & - \\
\hline
\end{tabular}

Keys: Post-infection (PI), Mild lesion (+), Moderate lesion (++), Severe lesion (+++) and No significant gross pathological lesion (-). Group B (Uninfected-untreated) is the control

Table 1: Gross lesions in donkeys experimentally infected with Trypanosoma evansi 
Group B (Uninfected, untreated): The various organs of the control animals (Uninfected, untreated) showed organs with normal colour, architecture and texture from post-mortem conducted at termination of experiment on day 176 PI (Table 1, Plates Ia \& IIa).
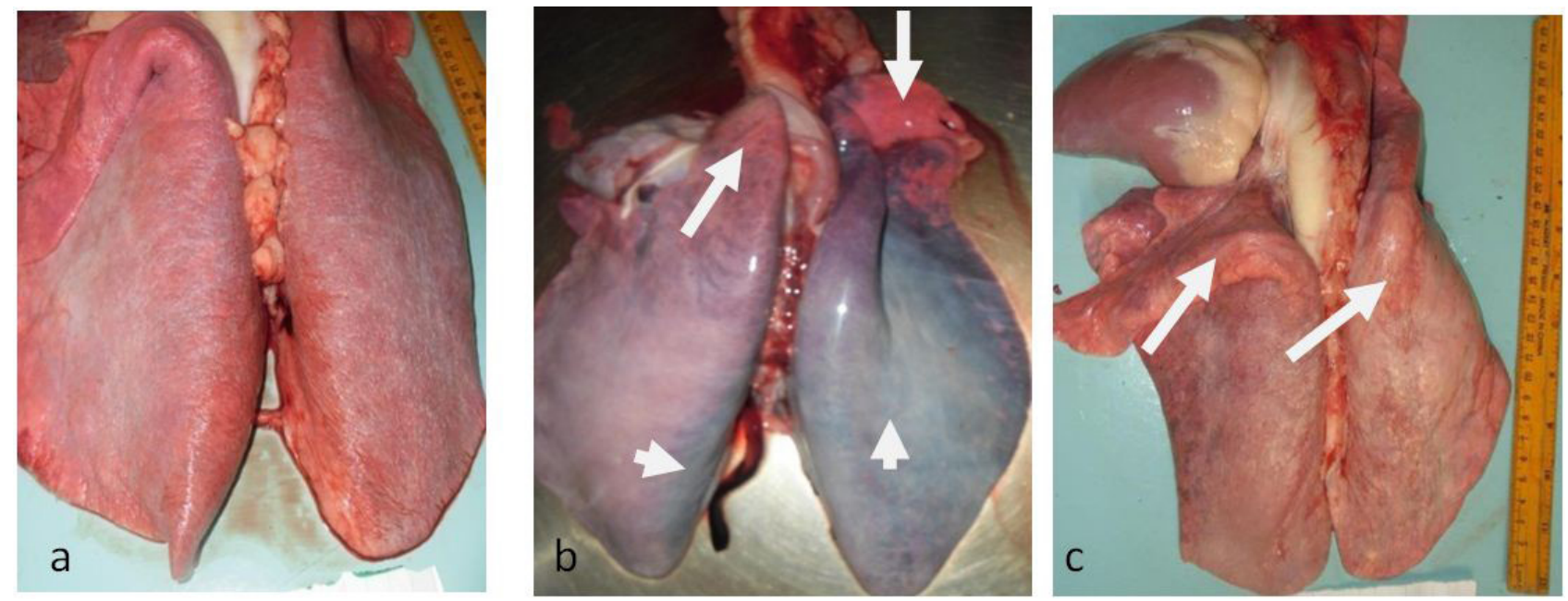

Plate I: Lungs of donkeys. Normal gross features in Uninfected-untreated (control) donkey at termination of experiment (a), congestion (arrowed) and gray hepatization (arrow heads) in T. evansi-infected, untreated donkey on day28 PI (b) and congestion (arrowed) in a T. evansi-infected, isometamidium-treated donkey on day 148 post-treatment

\section{Histopathological lesions observed in the Trypanosoma evansi-infected donkeys}

Histopathological examination of tissue sections revealed significant lesions only in the lungs, spleen and liver of infected animals from the postmortem performed on day 176 post-infection; equivalent to day 148 post-treatment. Only plates that show significant lesions (for infected groups) are presented in this study (Table 2).

\begin{tabular}{|c|c|c|c|c|}
\hline \multirow{2}{*}{$\begin{array}{c}\text { Organs } \\
\text { showing } \\
\text { lesions }\end{array}$} & \multirow[b]{2}{*}{ Lesions observed } & \multicolumn{3}{|c|}{ Severity of lesions in experimental group of donkeys } \\
\hline & & $\begin{array}{l}\text { Group A } \\
\text { (Infected- } \\
\text { untreated) }\end{array}$ & $\begin{array}{l}\text { Group } A_{2} \text { (In- } \\
\text { fected, Isomet- } \\
\text { treated) }\end{array}$ & $\begin{array}{c}\text { Group B } \\
\text { (Uninfected- } \\
\text { untreated) }\end{array}$ \\
\hline \multirow[b]{2}{*}{ Lungs } & -Congestion & +++ (Plate. IIIb) & - (Plate. IIIc) & - (Plate IIIa) \\
\hline & $\begin{array}{l}\text { Mononuclear cel- } \\
\text { lular infiltration }\end{array}$ & +++ (Plate. IIIb) & ++ (Plate. IIIc) & - (Plate IIIa) \\
\hline \multirow[b]{2}{*}{ Spleen } & -Haemosiderosis & +++ (Plate. IVb) & + (Plate. IVc) & - (Plate. IVa) \\
\hline & $\begin{array}{l}\text { Depletion of } \\
\text { lymphocytes at ger- } \\
\text { minal centres }\end{array}$ & +++ (Plate. IVb) & $+($ Plate. IVc) & - (Plate. IVa) \\
\hline \multirow{3}{*}{ Liver } & -Congestion & +++ (Plate. Vb) & - & - (Plate. Va) \\
\hline & $\begin{array}{c}\text {-Focal areas of } \\
\text { necrosis of hepato- } \\
\text { cytes }\end{array}$ & ++ (Plate. Vb) & - & - (Plate. Va) \\
\hline & $\begin{array}{l}\text {-Mononuclear cel- } \\
\text { lular infiltrations }\end{array}$ & $++($ Plate. Vb) & - & - (Plate. Va) \\
\hline
\end{tabular}

Keys: ${ }^{\star}$ Isomet $=$ isometamidium, Group B $=$ control. ${ }^{\star}$ Mild lesion $(+)$, Moderate lesion $(++)$, Severe lesion $(+++)$ and No significant histopathological lesion (-)

Table 2: Histopathological lesions observed in the Trypanosoma evansi-infected donkeys with and without treatment on day 176 post-infection (day 148 post-treatment

Group $\mathbf{A}_{1}$ (Infected-untreated): The histopathological lesions observed in this group of animals were severe forms of lungs congestion and mononuclear cellular infiltration (Table 2, Plate IIa), Splenic haemosiderosis and depletion of lymphocytes at germinal centres (Table 2, Plate IVb) and liver congestion (Table 2, Plate Vb). The liver sections also showed moderate levels of focal areas of hepatic necrosis and mononuclear cellular infilteration (Table 2, Plate $\mathrm{Vb}$ ).

Group $\mathbf{A}_{2}$ (Infected, isometamidium-treated): The animals treated with Isometamidium chloride, showed moderate levels of mononuclear cellular infiltrations in lungs (Table 2, Plate $\mathrm{Vb}$ ) and mild forms of splenic haemosiderosis and depletion of lymphocytes at germinal centres (Table 2, Plate IVc)

Group B (Uninfected, untreated): The control animals (Uninfected, untreated) showed normal histopathological features in all sections of organs examined (Table 2, Plate IIIa, IVa \& Va). 

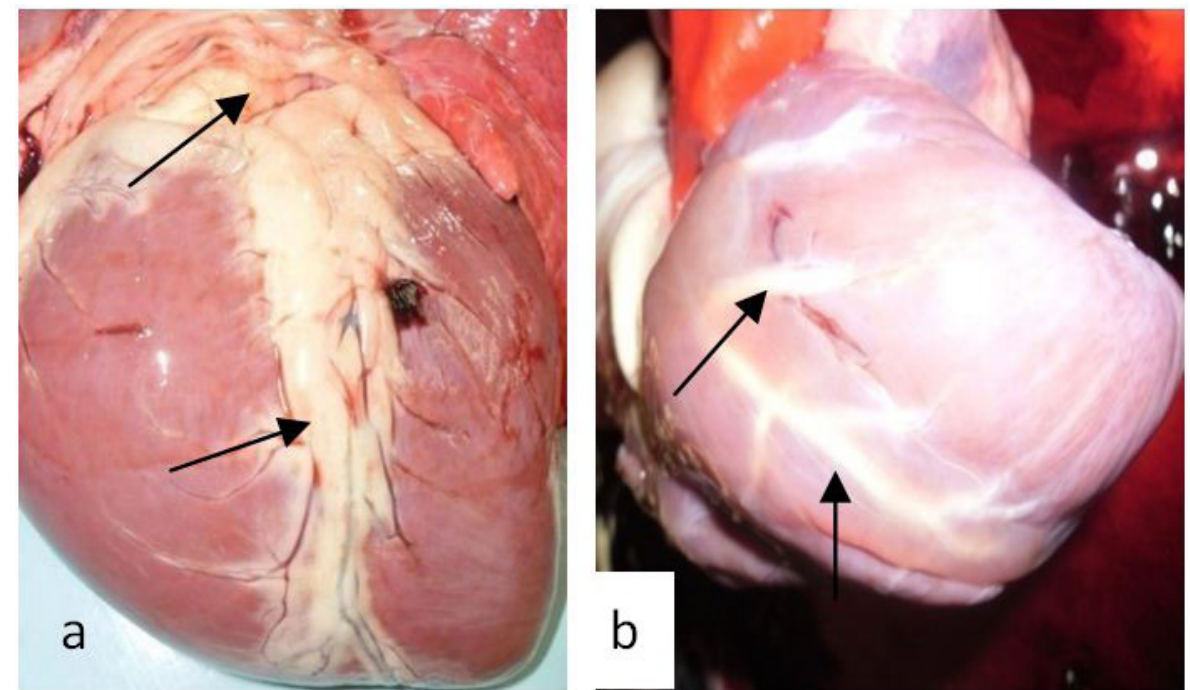

Plate II: Heart of donkeys. Intact coronary fat (arrowed) from an Uninfected-untreated (control) donkey at termination of experiment (a) and serous atrophy of coronary fat (arrowed) in T. evansi-infected, untreated donkey on day 176 post-infection (b)
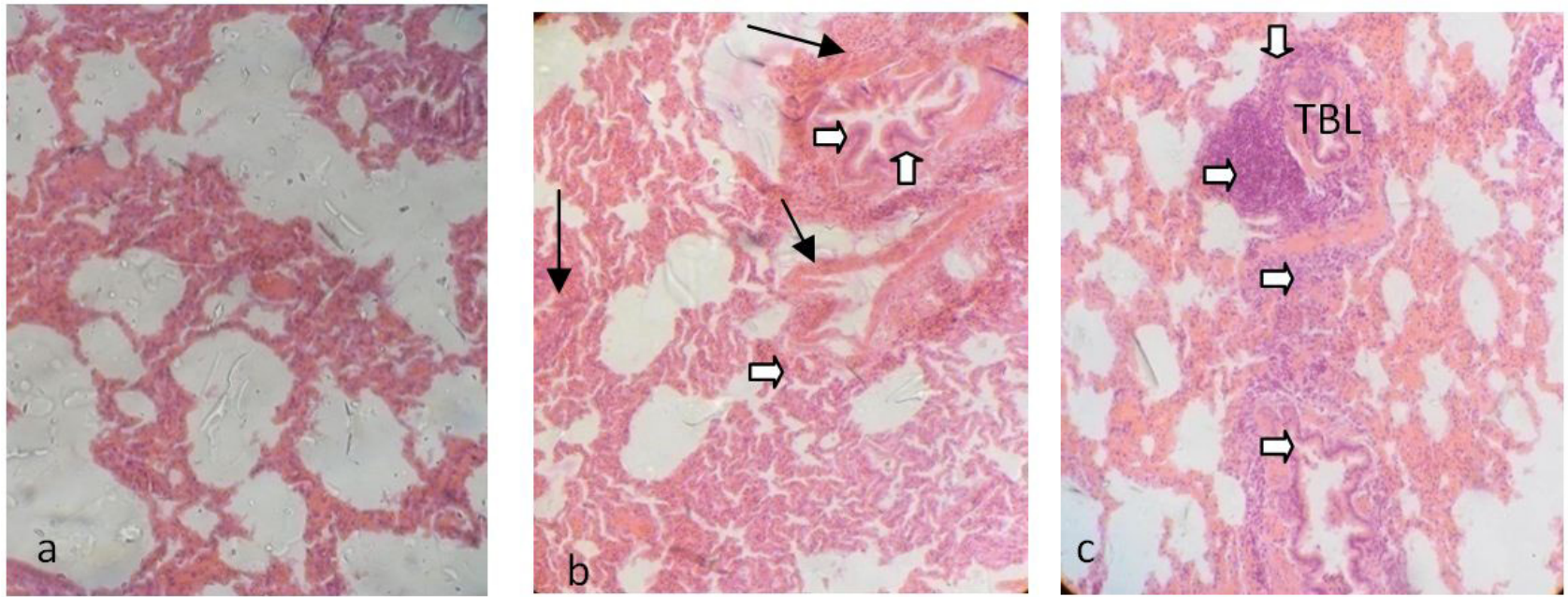

Plate III: Histopathological sections of lungs of donkeys. Normal section of an uninfected-untreated (control) donkey (a), congestion (arrowed) and mononuclear cellular infiltration (arrow heads) in T. evansi-infected but untreated donkey as at day 176 PI (b) and mononuclear cellular infiltrations (arrow heads) around terminal bronchiole (TBL) in T. evansi-infected but isometamidium-treated donkey as at day148 PI (c). Haematoxylin-Eosin (H-E) stain x200
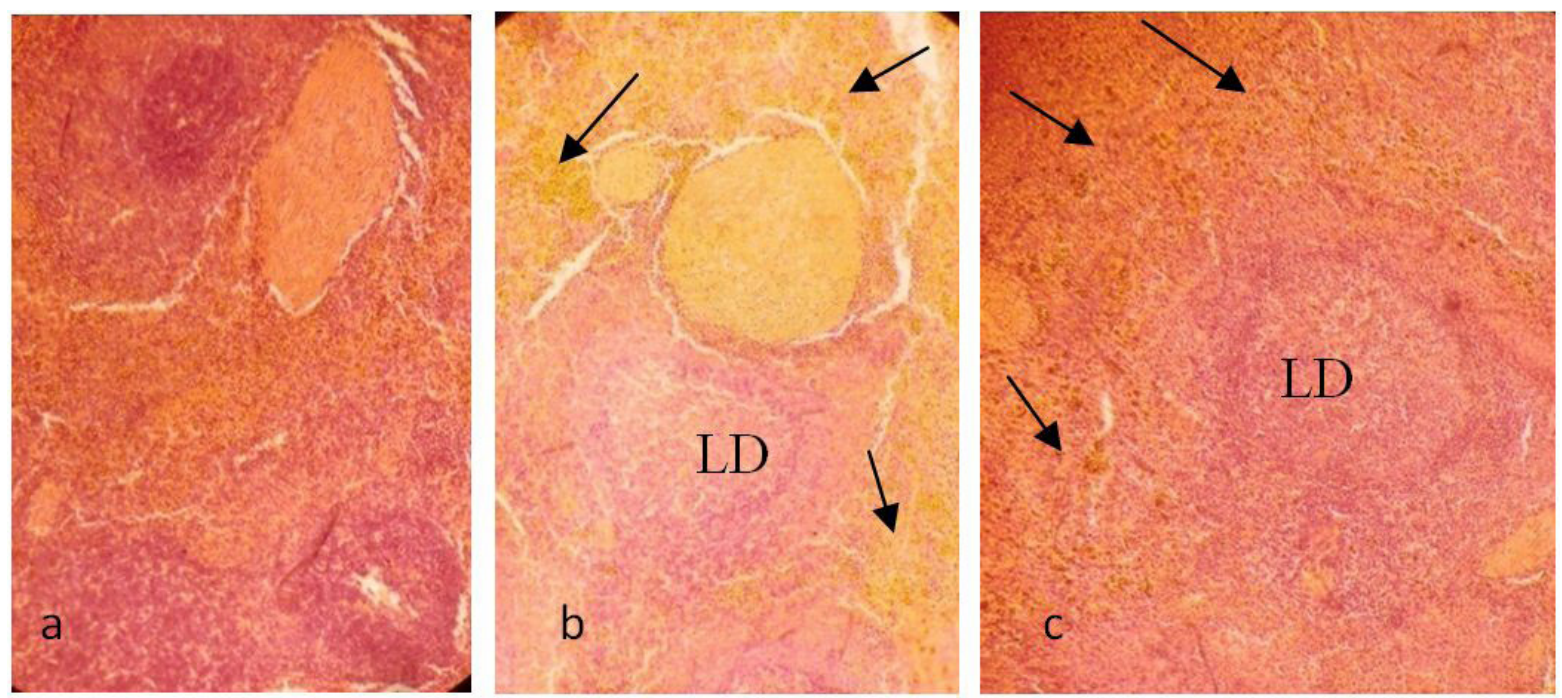

Plate. IV: Histopathological section of spleen of donkeys. Normal spleen section of Uninfected-untreated (control) donkey (H-E, x400) (a), haemosiderosis (arrowed) and lymphocytes depletion at germinal centres (LD) in T. evansi-infected but untreated donkey as at day $176 \mathrm{PI}$ (H-E, x200) (b) and haemosiderosis (arrowed) and lymphocytes depletion at germinal centres (LD) in T. evansi-infected but isometamidium-treated donkey as at day 148 post-treatment (H-E, x400) (c) 


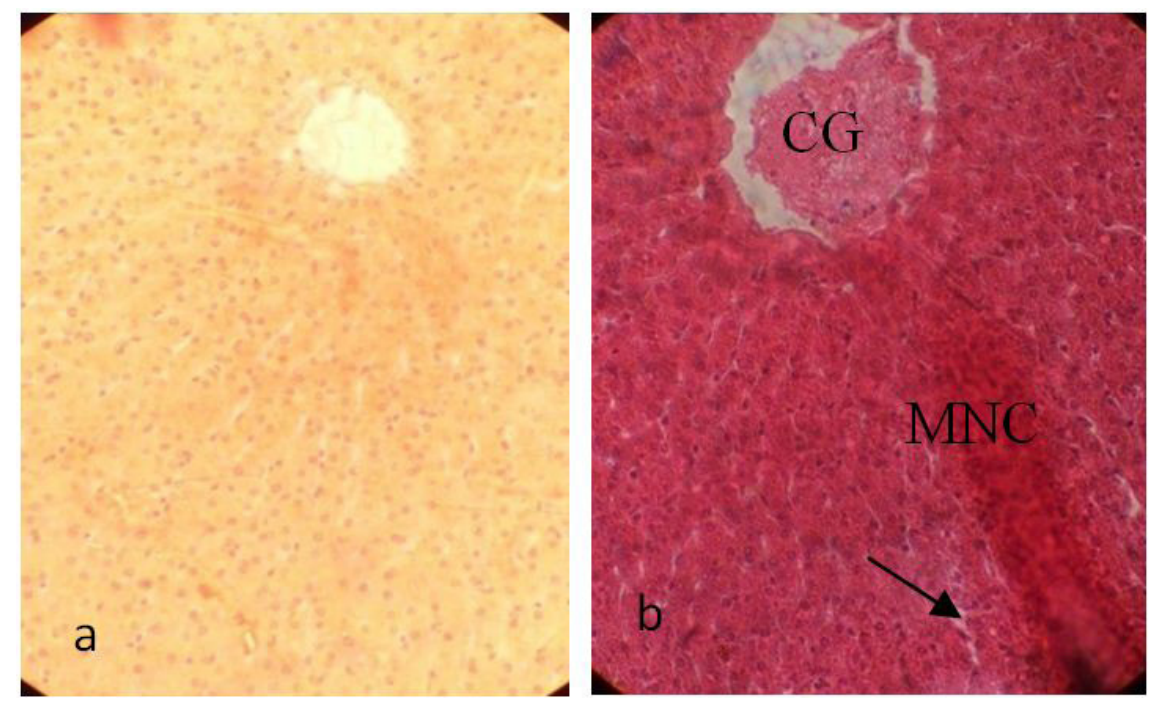

Plate V: Histopathological sections of the liver of donkeys. Normal liver section of Uninfected, untreated (control) donkey (a), and congestion (CG), focal necrosis (arrowed) and mononuclear cellular infiltration (MNC) in T. evansi-infected-untreated donkey as at day 148 post-treatment (b). H-E, x400

\section{Discussion}

In this study, the pre-patent period (PPP) of experimental T. evansi infection in donkeys was 3 days. This finding is earlier by a day than the 4 to 13 days reported earlier for most domestic animals by Ramirez et al. [31]. This means that the donkeys in this experiment are either more susceptible or the T. evansi isolate is more virulent than reported from earlier study. The $100 \%$ morbidity showed that T. evansi (Sokoto isolate) originally isolated from naturally infected camel, is pathogenic to donkeys. In related studies, strains of T. evansi have been reported to be pathogenic to donkeys in other parts of the world [32,33]. The low acute mortality rate in infected animals, chronic latent parasitaemia and parasites relapse in infected-untreated animals, survival of infected animals beyond the day of termination of experiment suggest that the course of surra in donkeys is predominantly chronic. This finding in Nigeria, agrees with earlier reports from other parts of the world that surra in donkeys is mainly chronic by Faye et al. [34] and Desquesnes et al. [33].

The parasitaemia levels in peripheral blood of infected animals fluctuated in early stage of infection and disappeared in chronic stage. This trend of parasitaemia pattern in the donkeys, suggests trypanosomes ability to reside intermittently in intra- or extravascular fluids of the donkeys as reported by Sudarto et al. thereby lowering parasitaemia in peripheral blood which made microscopic detection in later stage of infection difficult [35-37]. Trypanosomes also switch their surface glycoprotein to evade host immune responses resulting in relapses of parasitaemia and remittent clinical signs as reported by Hutchinson et al. [38]. The latent parasitaemia status detected chronically in the Infected-untreated group using mice inoculation test (MIT), and the death (due to high parasitaemia) of all mice used suggest that infected-untreated donkeys, assume chronic clinical or subclinical carriers status with low level but virulent and pathogenic T. evansi [21]. Mice inoculation test is reported to be 50 to $74 \%$ more sensitive than direct microscopic methods (e.g HCT) and it detects latent parasitaemia which explains the ability of MIT to detect latent parasitaemia in the present study [39]. The isometamidium-treated group showed negative parasitaemia status in the first 50 days post-treatment (PT) but later $60 \%$ of treated animals relapsed on MIT on day 100 with no death of inoculated mice recorded. It can be said that isometamidium chloride did not completely remove T. evansi infection from the treated donkeys but the remaining parasites were rendered non-pathogenic and avirulent. This finding might be due to resistance of T. evansi via switching of its surface glycoprotein coat despite the reported ability of isometamidium to cleavage trypanosomes kinetoplast Deoxyribonucleic acid-topoisomerase (kDNA-topoisomerase) complexes $[38,40]$ and causing disintegration of minicircle network within the $T$. evansi kinetoplast via mechanisms that are independent of kDNA to cause parasite death [41].

The gross pathological lesions observed in the infected-untreated group of animals were limited to the lungs in acute stage and comprised of only severe forms of frothy exudates in trachea and bronchi as well as lungs congestion. This finding agrees with earlier report by Cadioli et al. that donkeys may not show gross lesions in the acute stage of T. evansi infection [42]. The reduction in severity of lungs lesion to the mild form in later stage, as well as the emergence of moderate forms of hydrothorax, hydroperitoneum and adherence of renal capsule to the renal cortex suggests reduction in parasitaemia level but the increased number of gross lesions in the chronic stage points to the fact that the disease in donkeys, predominantly runs a chronic course [21,42].

The infected-isometamidium-treated animals did not show persistence of the lungs lesion however, there was emergence of moderate forms of gray hepatisation, hydrothorax and hydroperitoneum while adherence of renal capsule to the cortex was only mild, suggesting clearance or decreased parasitaemia. This might have been made possible because isometamidium chloride is a standard trypanocide reported to cure infection [12]. 
Journal of Veterinary Science \& Animal Husbandry

The histopathological findings in T. evansi-infected, untreated group of animals at termination of this experiment on day 176 PI (chronically) were severe forms of lung congestion and mononuclear cellular infiltration due to vascular stasis in lungs and liver, splenic haemosiderosis due to deposits of free iron from severe intravascular hemolysis, RBC sequestration in the red pulp area as reported by $\mathrm{Bal}$ et al. and cells lysis by T. evansi lymphotoxins also contribute to iron deposits in tissues [43,44]. Depletion of lymphocytes at germinal centres, severe hepatic congestion, the moderate forms of hepatic focal areas of necrosis and mononuclear cellular infiltrations the prominent lesions also reported from other parts of the world [43]. In the T. evansi-infected, but treated with isometamidium chloride, the chronic lesions were minimal and comprised of moderate form of pulmonary mononuclear cellular infiltration, mild forms of splenic haemosiderosis and depletion of lymphocytes at germinal centres. These decreases in lesions and their severities between untreated and treated animals can be explained first as due to reduced parasitaemia following treatment with isometamidium which is a standard trypanocides [12]. While the incomplete resolution of lesion may be due to $T$. evansi relapse as reported in camels treated using melarsenoxide cysteamine 4 months after the treatment [32].

\section{Conclusion}

It was concluded that in experimental T. evansi infection in donkeys, gross and histopathological lesions, ranging from severe to moderate forms are pronounced in the acute and chronic stages of infection involving the thoracic and peritoneal cavities, lungs, spleen, heart, kidneys and liver of affected animals that do not get treatment. Donkeys treated with isometamidium chloride show relapsed parasitaemia, mild to moderate histopathological lesions in lungs and spleen in the chronic stage with no gross lesions.

\section{Acknowledgement}

The contributions of the technical staff of Necropsy Unit and Veterinary Pathology Laboratory during post mortem examination and slides preparation respectively are highly appreciated.

\section{Ethical considerations}

The housing and care of the experimental animals as well as procedures performed on them were according to the guidelines on accommodation and care for animals used for experiment and other scientific purposes of the European Communities, contained in Appendix-A of Council Directive 86(609) EEC, 'Directive 2010/63' and approved by Research and Ethics Committee of Ahmadu Bello University, Zaria, Nigeria.

\section{References}

1. Dia ML, Diop C, Aminetou M, Jacquiet P, Thiam A (1997) Some factors affecting the prevalence of Trypanosoma evansi in camels in Mauritania. Vet Parasitol 72: $111-20$.

2. OIE [Office International des Epizootes] (2004) Manual of diagnostic tests and vaccines for terrestrial animals [online]. Paris: OIE; Trypanosoma evansi infections (including surra).

3. Kirsten Gillingwater aus Hornussen AG (2007) Discovery of novel active diamidines as clinical candidates against Trypanosoma evansi infection. Inaugural dissertation, zur Erlangung der Würde einer Doktorin der Philosophie, vorgelegt der, Philosophisch-Naturwissenschaftlichen Fakultät, der Universität Basel, 2007, 229 pp.

4. World Health Organisation (2012) Trypanosomiasis, Human African sleeping sickness. Fact sheet No: 259. Retrieved May 01, 2013.

5. Luckins AG (1999) Trypanosomiasis caused by Trypanosoma evansi in Indonesia. J Protozool Res 8: 144-52.

6. Kumar R, Kumar S, Virmani N, Yadav SC (2015) Transplacental transmission of Trypanosoma evansi from experimentally infected donkey mare to neonatal foal. J Equine Vet Sci 35: 337-4.

7. Ilemobade AA (1971) Studies on the incidence and pathogenicity of Trypanosoma evansi in Nigeria. 11: The pathogenicity of Trypanosoma evansi for equine and bovine species. ISCTRC, OAU/STRC publication 105: 107-14.

8. International Livestock Research Institute Monograph (1997) Disease resistance and protecting the environment. In; Livestock, people and the environment $10-11$.

9. Da Silva AS, Zanette RA, Wolkmer P, Costa MM, Garcia HA, et al. (2009) Diminazene aceturate in the control of Trypanosoma evansi infection in cats. Vet Parasitol 165: 47-50.

10. FAO [Food \& Agricultural Organisation] (2012) A field guide for the diagnosis, treatment and prevention of African animal trypanosomiasis. Retrieved September 22, 2012.

11. Touratier L (1981) (The advantages of using Isometamidium for the control of animal trypanosomiasis). International Scientific Council for Trypanosomiasis Research and Control. Sixteenth meeting, Yaounde, Cameroon, 1979. OAU/STRC: 308- 16.

12. Aliu YO (2007) Nigerian Veterinary Formulary; Handbook of Essential Veterinary Drugs, Biologics and Pesticide Chemicals. First edition, Veterinary Council of Nigeria 67.

13. Mehlhorn H (2008) Encyclopedia of parasitology. (Third edition) Springer. Verlag, Heidelberg, Germany 1-2.

14. Delespaux V, De Koning HP (2007) Drugs and drug resistance in African trypanosomiasis. Drug Resistance Update 10: 30-50.

15. Karaye GP (2012) The Efficacy of Isometamidium chloride In the Treatment of Trypanosomosis in Red Sokoto Bucks experimentally infected with Trypanosoma congolense and Trypanosoma brucei single and mixed Infection of The Two. MSc. (Veterinary sciences) thesis submitted to the School Of Postgraduate Studies, Ahmadu Bello University, Zaria, Nigeria.

16. Wayne L, Melvin B (2000) Determining age of horses by their teeth, Extension and Agricultural Information. Agriculture Building, Columbia, MO 65211; XPLOR 1-98.

17. Joe A (2012) The Donkey Sanctuary. British Veterinary Association. 
18. Pearson RA, Ouassat M (2000) A Guide toLivestock Weight Estimation and Body Condition ofDonkeys. Centre for Tropical Veterinary Medicine, University of Edinburgh, UK.

19. Food \& Agricultural Organisation (2014) Animal production: Section 1, Modul 5, Livestocks Systems Research Manual-Volume 1. Produced by ILRI. Retrieved December 16, 2014.

20. De Aluja AS1, Tapia Pérez G, López F, Pearson RA (2005) Liveweight estimation of donkeys in central mexico from measurements of thoracic circumference. Trop Anim Health Prod 1: 159-71.

21. Office International des Epizootes (2010) Trypanosoma evansi infection (surra).Version adopted by The World Assembly of Deligates of the OIE.

22. Wolfensohn S, Lloyd M (2013) Handbook of Laboratory Animal Management and Welfare (4th edn), Wiley-Blackwell Publishing ltd, UK.

23. Aviva P, Poul W (2013) Statistics for Veterinary and Animal Science (3rd edn), Wiley-Blackwell ltd, UK.

24. Aganga AA, Letso M, Aganga AO (2000) Feeding donkeys. Livestock Research for Rural Development 12.2.

25. Charles MH (2007) Internal parasites, External paraspites. In Charles M, Margi S (Eds) Laboratory procedures for veterinary technicians. 5th Edn, Mosby Elsevier Inc, St Louis, Missouri, 187-252.

26. Elaine A, Margi S (2007) Hematology and hemostasis. In Charles MH, Margi S (Eds) Laboratory Procedures for Veterinary Technicians (5th edn), Mosby Elsevier Inc, St Louis, Missouri, 27-73.

27. Weiser G (2012) Laboratory technology for veterinary medicine, In Mary AT, Glade W, Robin WA, Terry WC (eds) Veterinary Hematology and Clinical Chemistry, second edition, Wiley-BlackWell, UK 3-33.

28. Monzon CM, Mancebo OA, Roux JP (1990) Comparison between 6 parasitological methods for diagnosis of Trypanosoma evansi in the subtropical area of Argentina. Vet Parasitol 36: 141- 6.

29. Woo PT (1970) The haematocrit centrifugation technique for the diagnosis of African trypanosomiasis. Acta Tropica 27: 384-6.

30. Drury RAB, Wallington EA (1976) Carteton's Histological techniques (4th edn), Oxford University Press, London 21-70.

31. Ramirez LE, Wells EA, Betancourt A (1979) La trypanosomises en los animales domesticos en Colombia (S.L.) Centro International Agricultura Tropical 71.

32. Berlin D, Nasereddin A, Azmi K, Ereqat S, Abdeen Z, et al. (2010) Longitudinal study of an outbreak of Trypanosoma evansi infection in equids and dromedary camel in Israel. Vet Parasitol 174: 317-22.

33. Desquesnes M, Holzmuller P, Lai D, Dargantes A, Lun Z, et al. (2013) Trypanosoma evansi and surra: A review and perspective on origin, history, distribution, taxonomy, morphology, host and pathogenic effects. 2013, ID-194176, 22pp.

34. Faye D, Pereira de Almeida PJ, Goossens B, Osaer S, Ndao M, et al. (2001) Prevalence and incidence of trypanosomosis in horses and donkeys in The Gambia. Vet Parasitol 101: 101-14.

35. Sudarto MW, Tabel H, Haines DM (1990) Immunohistochemical demonstration of Trypanosoma evansi in tissues of experimentally infected rats and a naturally infected water buffalo (Bubalus bubalis). J Parasitol 76: 162-7.

36. Pinchbeck GL, Morrison LJ, Tait A, Langford J, Meehan L, et al. (2008) Trypanosomosis in The Gambia: Prevalence in working horses and donkeys detected by whole genome amplification and PCR, and evidence for interaction between trypanosomes species. Biomed Central Vet Res 48: 4-7.

37. Muieed MA, Chaudhary ZI, Shakoori AR (2010) Comparative study on the sensitivity of PCR AND ME for the detection of Trypanosoma evansi in horses. Turkish J Vet Anim Sci 34: 507-12.

38. Hutchinson OC, Picozzi K, Jones NG, Mott H, Sharma R, et al. (2007) Variant Surface Glycoprotein gene repertoires in Trypanosoma brucei have diverged to become strain-specific. BMC Genomics 8: 234.

39. Luckins AG (2014) Methods for the diagnosis of trypanosomiasis in livestock. Centre for Tropical Veterinary Medicine, Eat Bush, Roslin, Midlothion EH25 9RG, UK.

40. Shapiro TA, Englund PT (1990) Selective cleavage of kinetoplast DNA minicircles promoted by antitrypanosomal drugs. Proc of the Natl Acad Sci USA 87: 950-4.

41. Kaminsky R, Schmid C, Lun ZR (1997) Susceptibility of dyskinetoplastic Trypanosoma evansi and Trypanosoma equiperdum to isometamidium chloride. Parasitol Res 83: 816-8.

42. Cadioli FA, Marques LC, Machado RZ, Alessi AC, Aquino LPCT, et al. (2006) Experimental Trypanosoma evansi infection in donkeys: Haematological, biochemical and histopathological changes. Arquivo Brasileiro de Medicina Veterinaria e Zootecnia.

43. Bal MS, Singla LD, Kumar H, Vasudev A, Gupta K, et al. (2012) Pathological studies on experimental Trypanosoma evansi infection in Swiss albino mice. J Parasitic Dis 36(2): 260-64

44. Antoine-Moussiaux N, Cornet A, Cornet F, Glineur S, Dermine M, et al. (2009) A Non-Cytosolic Protein of Trypanosoma evansi Induces CD45-Dependent Lymphocyte Death. PLoS ONE 4: e5728.

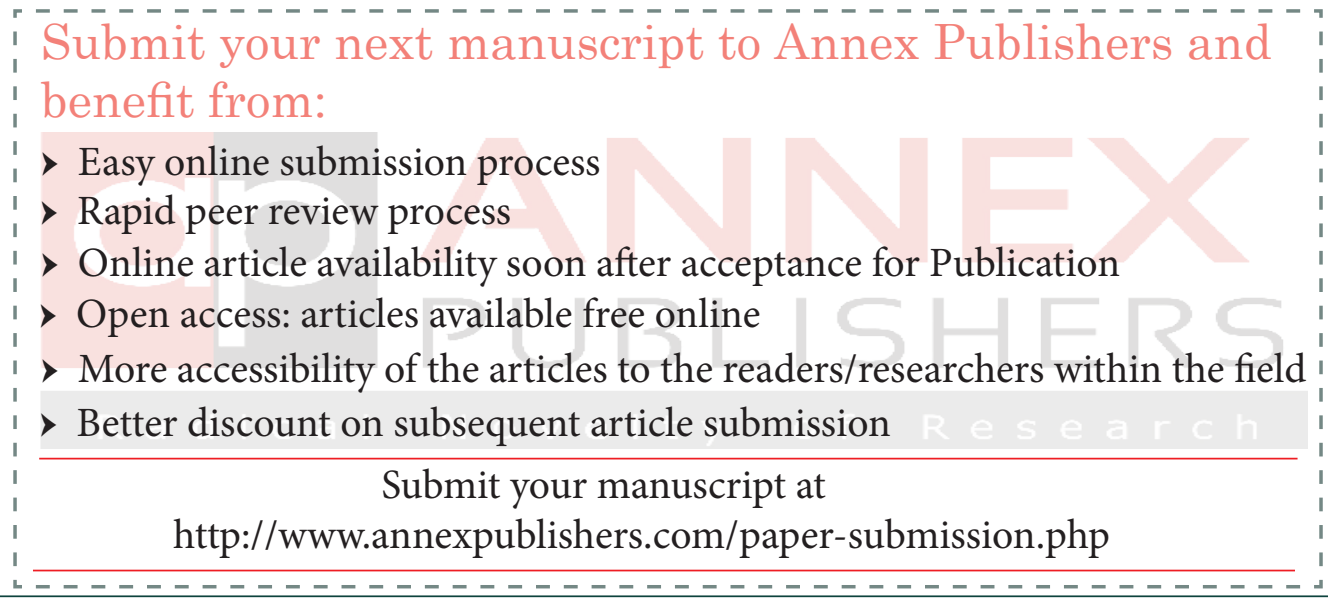

